\title{
Control Methods in Microspheres Precision Assembly for Colloidal Lithography
}

\author{
Olivier Delléa ${ }^{1}$, Olga Shavdina ${ }^{1}$, Pascal Fugier ${ }^{1}$, Philippe Coronel ${ }^{1}$, \\ Emmanuel Ollier ${ }^{1}$, and Simon-Frédéric Désage ${ }^{2}$ \\ ${ }^{1}$ L2CE, Laboratoire des Composants pour le Conversion de l'Energie, CEA/LITEN, \\ Laboratoire d'Innovation pour les Technologies des Energies Nouvelles et des nanomatériaux, \\ Grenoble, France \\ ${ }^{2}$ SYMME, Laboratoire des Systèmes et Matériaux pour la Mécatronique, Université de Savoie, \\ Annecy, France
}

\begin{abstract}
Colloidal lithography based on the assembly of microspheres is a powerful tool for the creation of a large variety of two dimensional micro or nanostructures patterned. However very few studies examine the control, qualification and quantification of the ordering of the particles once deposited on the substrate. We have developed two unique methods working at microscopic and macroscopic scales, respectively called Microfixe ${ }^{\circledR}$ and Macrofixe ${ }^{\circledR}$, for the analysis of grain morphology in the case of hexagonal closed packed (HCP) monolayers of spherical microparticles. The processing of the images taken at microscopic scale uses Delaunay triangulation and histograms of lengths and orientations of Delaunay triangles sides. At the macroscopic scale, six camera images are required of the sample illuminated under six different incidence angles. Image treatment consists in the comparison of the six images and eventually subdivision of these images to sharpen the analysis. At the end, the two softwares constitute artificial images of particle deposit, giving at microscopic and macroscopic scales significant information about grain size, grain morphology, orientation distributions, defaults (voids, stacking)... With these two new control methods, colloidal lithography is emerging as an industrial process.
\end{abstract}

Keywords: Metrology, Quality Control, Computer Vision, Image Processing. Quad tree, Microfixe ${ }^{\circledR}$, Macrofixe ${ }^{\circledR}$, Colloidal lithography, micro/nanotechnologies, thin films, tribology, BooStream ${ }^{\circ}$.

\section{Introduction}

Colloidal lithography based on the precision assembly of microspheres is an easy, inexpensive, efficient, and flexible manufacturing approach [1]. It enables the creation of a large variety of two dimensional micro or nanostructures patterned with a high degree of control and reproducibility [2]. Many application areas are concerned by this technology such as mechanics [3], sensors, photonics, surface wetting [4], biological and chemical sensing. One can find many studies aiming at the 
self-assembly control or ordering of particles acting on the physical processes such as activation of particle surfaces, solvents, surface pressure... but very few of them examine the control, qualification and quantification of the ordering of the particles once deposited on the substrate.

In the case of silica microspheres, the inherent 2D periodicity constituted by a hexagonal-close-packed (HCP) type ordering (Figure 1) gives rise to a rich variety of interesting optical properties related with photonic crystals.

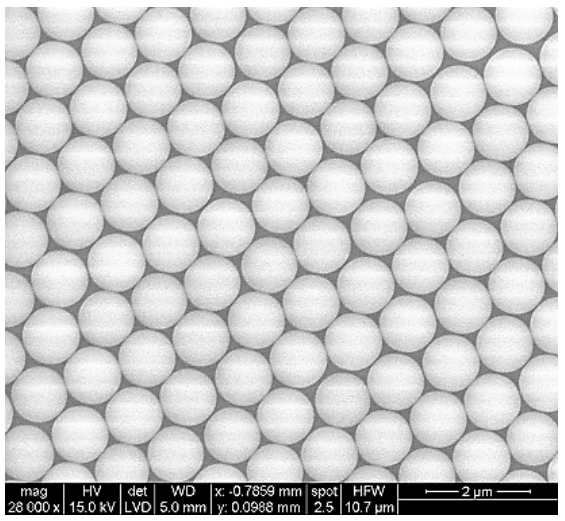

Fig. 1. Hexagonal-close-packed (HCP) monolayer of silica microspheres $\varnothing 1.1 \mu \mathrm{m}$

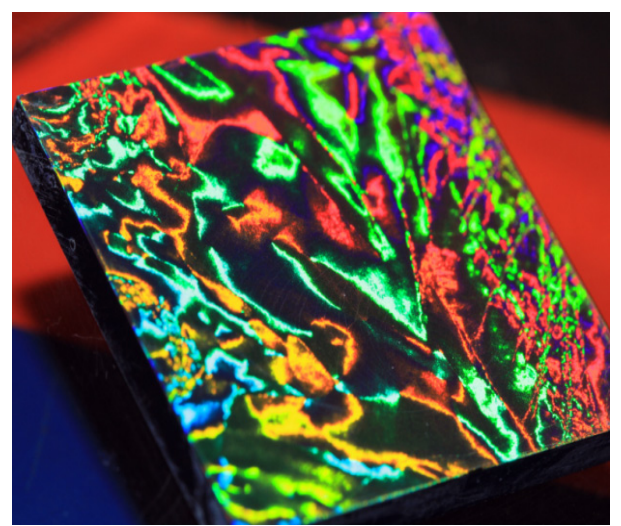

Fig. 2. Color response of an HCP monolayer of silica microbeads $\varnothing 1.1 \mu \mathrm{m}$ on diamond like carbon substrate $2 \times 2 \mathrm{~cm}^{2}$

As a result of photonic band gap properties, the 2D-ordered microstructure creates intensive structural colors [5] [6] as shown in Figure 2. In fact, due to process parameters (particle size distribution, surface pressure, withdrawing speed...), the particle deposits are composed of "grains" [7] separated by "fractures". Each grain is composed of hundreds or thousands of particles forming a hexagonal mesh with a specific planar orientation (Figure 3).

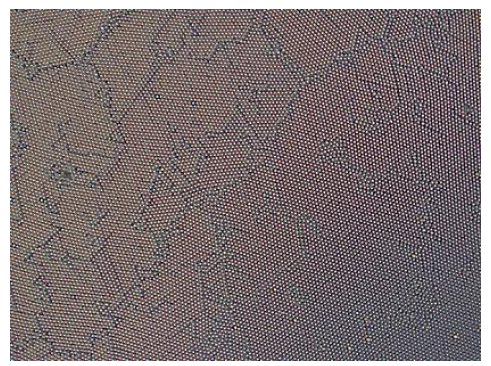

Fig. 3. Microscope image $(\mathrm{x} 100)$ of HCP silica microspheres $(\varnothing 1.1 \mu \mathrm{m})$ displaying grains separated by fracture lines 
In order to master and improve the process, it is essential to control/qualify/ quantify in a rigorous way the grain sizes, grain orientations, fractures, etc. of the particle deposits.

The following paragraphs briefly introduce the precision assembly technique of microspheres, developed by CEA LITEN called BooStream ${ }^{\circledR}$, to create an HCP monolayer. The fields of application are numerous and we present succinctly its using in the field of tribology. The two analysis methods developed by CEA LITEN and based on image processing and vision are then detailed. The first is software, named Microfixe ${ }^{\circledR}$, which addresses the analysis of images taken at microscopic scale with optical microscope or scanning electron microscope. The second method, called Macrofixe ${ }^{\circledR}$, analyses visual rendering of particle deposit (iridescence) by processing a sequence of camera images taken with various lighting conditions.

\section{HCP Particles Monolayer Assembly}

\subsection{Presentation}

Particle self-assembly methods at the air/liquid interface are mainly based on the minimization of free energy: the formation of the monolayer is mainly due to the energy level reduction at the air/liquid interface.

Particles then order themselves [8] under different phenomena, such as dipole moments, partial positive charges and hydrophobicity attraction. Common techniques presented in the scientific literature are the Langmuir-Blodgett method [9], vortical method [10] and floating-transferring method [11].

The Boostream ${ }^{\circledR}$ process has been developed on the basis of previous work for the production of active or passive components in the field of energy. In its basic functions, this process uses a moving liquid on which particles are dispensed (Figure 4). Brought by hydrodynamic forces to a transfer area using a slope, the particles are arranged as a film on the liquid. The substrate set previously in contact with the compact film of particles through a capillary bridge is then withdrawn to transfer the film.

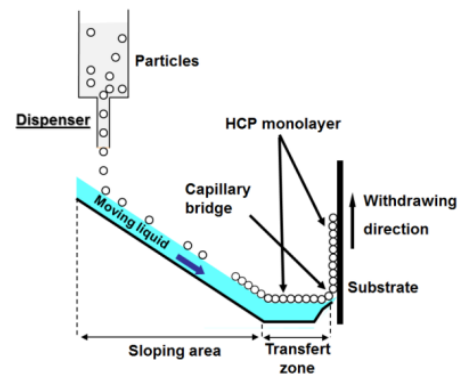

Fig. 4. The BooStream ${ }^{\circledR}$ process (basic configuration) 
In this basic configuration, the main advantage of this process is the ability to deal with online substrates of large surface, $2 \mathrm{D}$ or $3 \mathrm{D}$, rigid or flexible, for industrial applications.

\subsection{Example of Application: Tribology}

For several years, surface texturing has been introduced to improve tribological properties of lubricated surfaces [12]. The presence of artificially created microdimples on a frictional surface can generate substantial reduction in friction and surface damage when compared with non-textured surfaces. This improvement is attributed to several physical mechanisms like wear debris entrapment, the creation of local increase of lubricant supply by fluid reservoirs and also the increase of loadcarrying capacity by a hydrodynamic effect [13].

By combining colloidal lithography and oxygen plasma etching, textured diamondlike amorphous carbon (DLC) films can be produced. The process consists of depositing the monolayer of spherical particles on the DLC film and etching this layer with plasma through the hard mask of silica spheres, then to remove the particles from the substrate by immersion in water with ultrasonic waves. The DLC surface obtained by this method shows micro-pillar networks with dimensions that modulate the surface properties of this material (Figure 5).

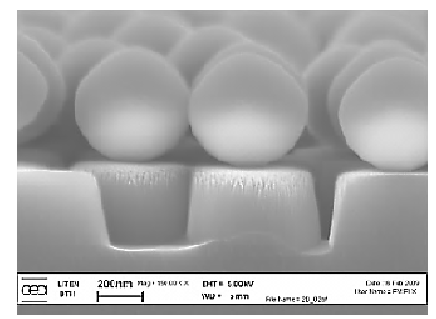

Fig. 5. Micro-pillar networks in DLC film with, on top, HCP silica particle deposit

By this technology, a reduction of $50 \%$ of the friction coefficient has been demonstrated in lubricated conditions [14].

At present, only a few mechanical components used in industrial applications are textured because each application requires an accurate correlation of the texture (depth, density, shape...) with the specific mechanical parameters (geometry, dimensions, load, speed, lubricant...).

This example demonstrates the importance of developing the following control processes described in this paper to apply this technology to industry.

\section{Control Method at Microscopique Scale : Microfixe ${ }^{\circledR}$}

\subsection{Image Processing Methodology}

Image processing is the main tool for the qualification/quantification of the number, size and orientation of the grains. A preliminary step is the extraction of the centre of 
each particle. Then a Delaunay triangulation [15] is done on this set of points, linking each point with its six (or less) neighbours. It will be useful in the sequel to consider that each link is materialized by a line segment. The orientation of these line segments (with a $60^{\circ}$ periodicity) is then fundamental.

A simple representation is obtained with the information given by the lengths and orientations of triangle histograms (Figure 6).

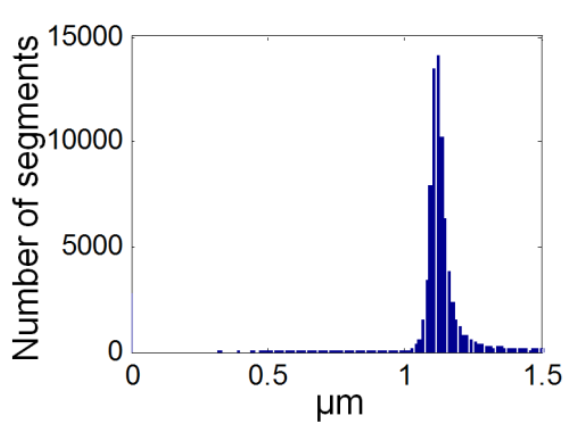

a) Lengths histograms.

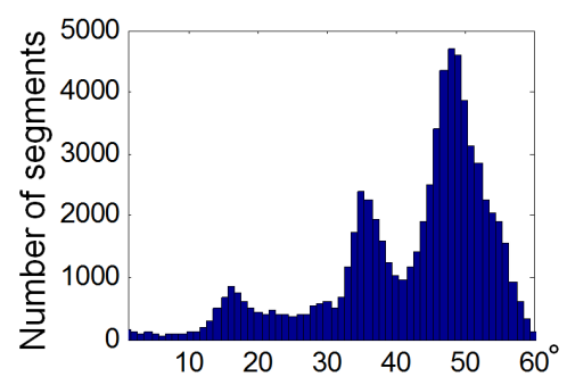

b) Orientation histograms.

Fig. 6. Analysis of Figure 3, histograms of Delaunay triangles segments

These histograms gather essential information for the determination of quality of the ordered deposits. If the histogram of the triangles' segments lengths distribution is centered on the value corresponding to the nominal particles average diameter, we can deduce that the deposit is compact with few fractures. Moreover, if the histogram of the sides of the triangles' orientations has a single mode (single peak) between $0^{\circ}$ and $60^{\circ}$, it means that the deposit of particles is composed of a single grain. In another way, if the triangles' lengths histogram has more than one peak, it means that the deposit of particles is fractured and composed of more than a single grain. In the example in Figure 6, the deposit has several grains with three main directions related to the three peaks on the histogram.

Furthermore, the Delaunay triangulation can be used for the calculation of the relative areas occupied by the different grains on the image giving an indication of the arrangement quality.

\subsection{Results}

Microfixe® software allows an accurate analysis of the quality of an HCP particles monolayer. Figure 7 shows two cases. The conditions of the deposition process are different in each case. In this example, two images taken with an optical microscope are used. The Delaunay triangulation of these two images is coloured in order to provide an enhancement of the deposit structure, assisting the monitoring of the process. 
Assuming that the maximization of grain size is the main criterion for the developed process, the calculation results clearly show that process $n^{\circ} 2$ overwhelms process $n^{\circ} 1$ with only three grains and two main orientations. The surface ratio between the ordered area, and the total area analyzed is also a thorough quality index, as shown by the table summarizing the results of Microfixe ${ }^{\circledR}$ software analysis.

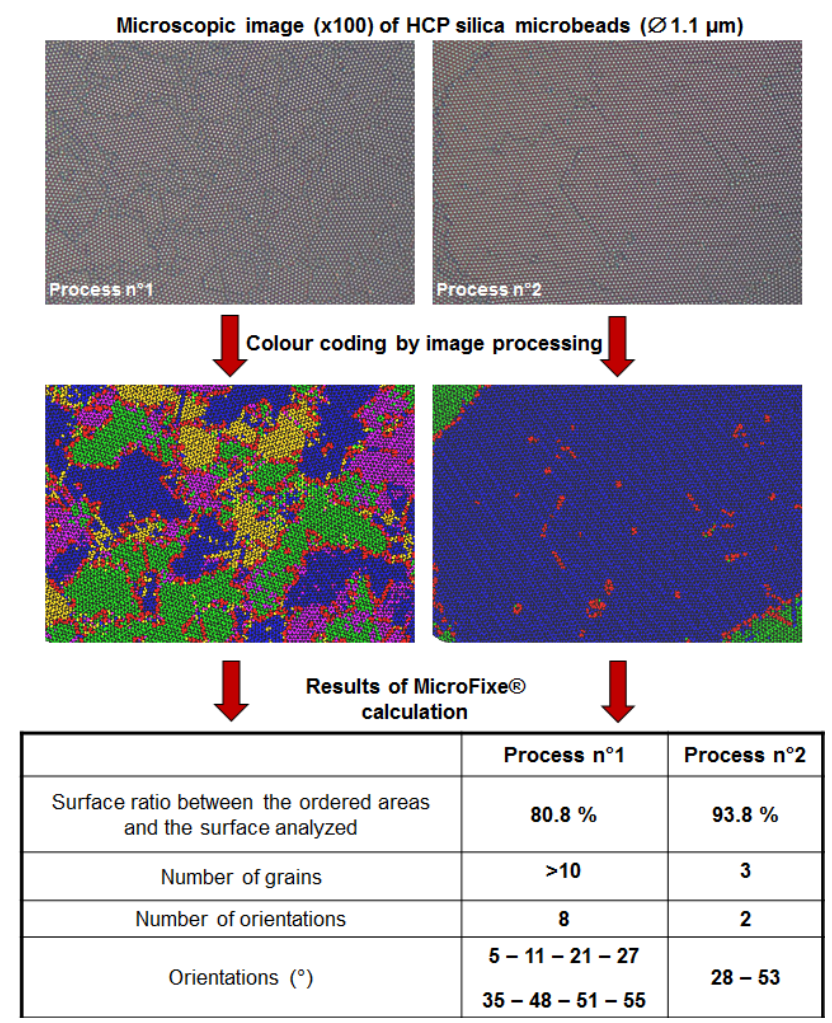

Fig. 7. Analysis of two deposits with Microfixe ${ }^{\circledR}$ software

\section{Control Method at Macroscopic Scale: Macrofixe ${ }^{\circledR}$}

Colour is a perceptual attribute created by the human brain in reaction to the stimulus of a certain light signal in a given context. At macroscopic scale, the grains forming part of the deposit have different colours due to the different orientations of the particle structure, which have influence on light diffraction, therefore on the visual appearance. In order to study the correspondence between particle structure and visual rendering, we can observe and analyze deposits with a RGB camera. 


\subsection{Structural Analysis by Multi-angle Optical Illumination}

The diffraction pattern of HCP deposited microspheres for a collimated light has a specific structure displaying symmetry. This pattern is composed of a specular reflection of light (zero order diffraction) surrounded, at equal distance, and distributed every $60^{\circ}$, by six diffracted beams at the first order of diffraction. The figure below illustrates the diffraction at the first order of a collimated beam having a wavelength $\lambda$ and incidence angles $\left(\theta_{\mathrm{i}}, \varphi_{\mathrm{i}}\right)$ on a screen positioned perpendicular to the propagation direction of the reflected beam $\left(\theta_{\mathrm{r}}, \varphi_{\mathrm{r}}\right)$.

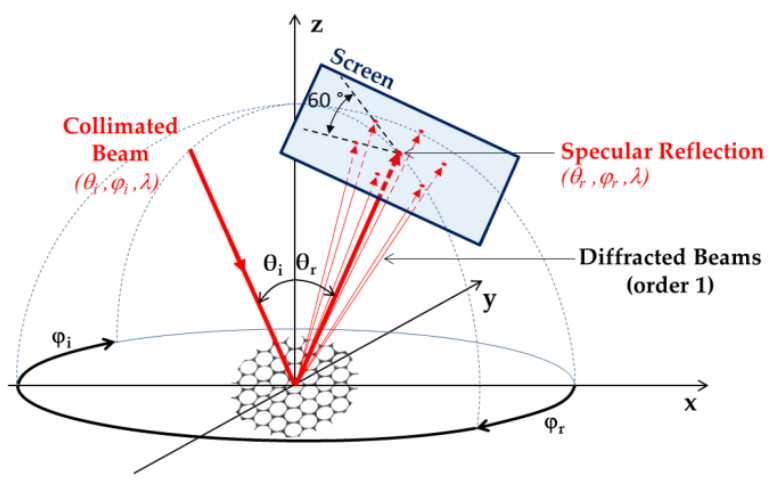

Fig. 8. Illustration of the diffraction phenomenon by HCP structure at zero and first order diffraction on a screen

This angle periodicity indicates that an angular variation of incident light with respect to the sample of $60^{\circ}$ in the $\mathrm{xOy}$ plane $\left(\varphi_{\mathrm{i}}\right)$ is enough to get diffracted towards a fixed point of observation on any HCP structure whatever its orientation in the xOy plane. On this basis, the Macrofixe ${ }^{\circledR}$ control method has been developed using only six illumination and/or observation positions with $10^{\circ}$ relative angle variations in the xOy plane $\left(\varphi_{\mathrm{i}}\right)$ to obtain the particle structure orientation in all grains.

The six images presented in Figure 9 are a view of the same sample captured from the same point of view, with six relative positions of the light source $\left(\varphi_{\mathrm{i}}=0,10^{\circ}\right.$, $20^{\circ}, 30^{\circ}, 40^{\circ}, 50^{\circ}$ ). This sample is a $10 \times 10 \mathrm{~mm}^{2}$ silicon substrate with HCP silica particles of $1.1 \mu \mathrm{m}$ diameter. The local colour of the sample is related to the orientation of incident light and to the particle orientation. In a given illumination-observation configuration, grains are coloured if they diffract light towards the observation point (camera), otherwise they appear black. Colour is thus a cartographical manner of indicating the local structure orientations of a large sample. 


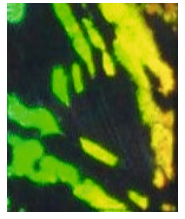

Image 1: $0^{\circ}$

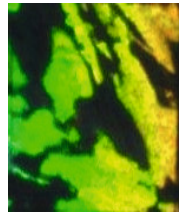

Image $2: 10^{\circ}$

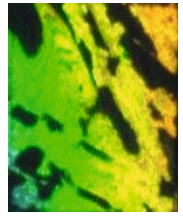

Image $3: 20^{\circ}$

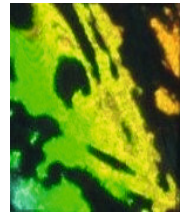

Image $4: 30^{\circ}$

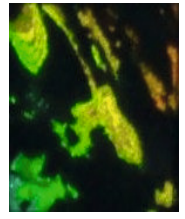

Image 5: $40^{\circ}$

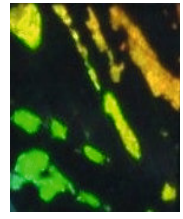

Image 6: $50^{\circ}$

Fig. 9. Images of the same silicon substrate sample $\left(10 \times 10 \mathrm{~mm}^{2}\right)$ with a deposit of $1.1 \mu \mathrm{m}$ diameter silica particles for six angle positions $\left(\varphi_{\mathrm{i}}\right)$ of a collimated white light source

Based on these six images, it is possible to extract a map of grains at macroscopic scale by image processing.

\subsection{Image Processing Methodology}

The six images in Figure 9 are first thresholded according to the method developed by Otsu [16] to create binary images. The percentage of white pixels of each image is then calculated. Figure 10 shows the dependence of the percentage of white pixels on six consecutive images. The amplitude between the min and max curve values is calculated. The greater the amplitude of the curve, the greater the observed structure can be considered as single crystal. In contrast, low amplitude shows that the optical response of the sample is relatively constant regardless of the lighting conditions. Therefore, the observed zone shows no particular structure and can be considered as scattering. Moreover, in the case where the amplitude is high enough, the image corresponding to the maximum of the curve indicates the main direction of diffraction and hence the orientation of the observed structure.

With this approach, it is therefore possible to map the grain structure but also to determine the orientation of the hexagonal structure for each of them.

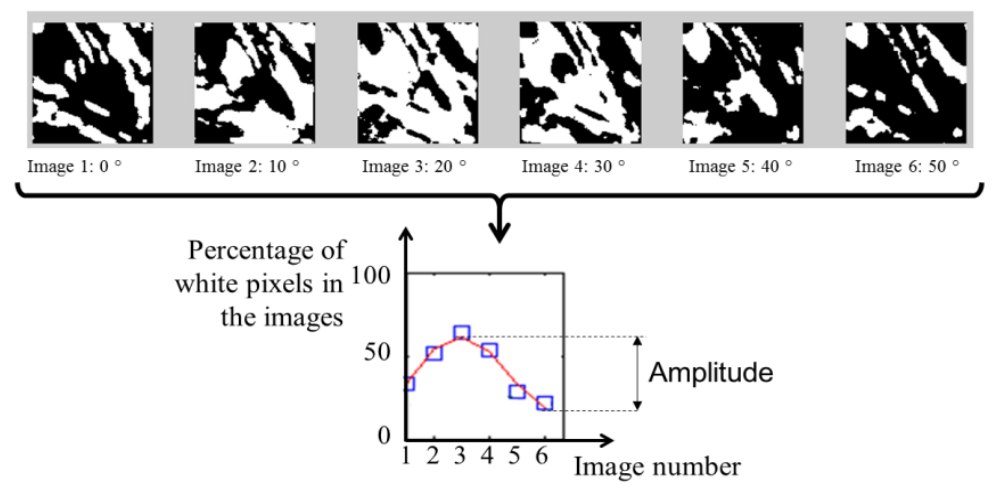

Fig. 10. View of binary images from Figure 9 and evolution curve of the white pixels percentage according to the six consecutive images 
In accordance with our criteria, in the case where the amplitude is in the range of $90 \%$ to $100 \%$, the observed area is formed by a single crystal (or near), the system can indicate a high quality of ordering. If the amplitude is between $0 \%$ and $20 \%$, and the observed area scatters the light, the particles are not organized. If the difference is between $20 \%$ and $90 \%$, the area cannot be characterized accurately and requires a more detailed analysis. Based on an analysis methodology called QuadTree [ ], each of the six images is then divided into four equal sectors. For each of the four areas an evolution curve of the white pixels number is performed. Similarly, the amplitude of each plotted curve is calculated to qualify characterise each division of the image. In the developed process, the subdivision of the image continues until a stop criterion is reached: ordered area, diffusing area, image size too small (only 1 pixel) etc.

\subsection{Results}

Based on the protocol described above, the Macrofixe ${ }^{\circledR}$ process is able to reconstitute an image of squares indicating the morphology of the grains and for each, its relative orientation in the plane of the hexagonal lattice. Figure 11 shows for example the treatment of images presented in Figures 9 and 10. The latter shows a large number of grains and many changes of the hexagonal lattice orientations.
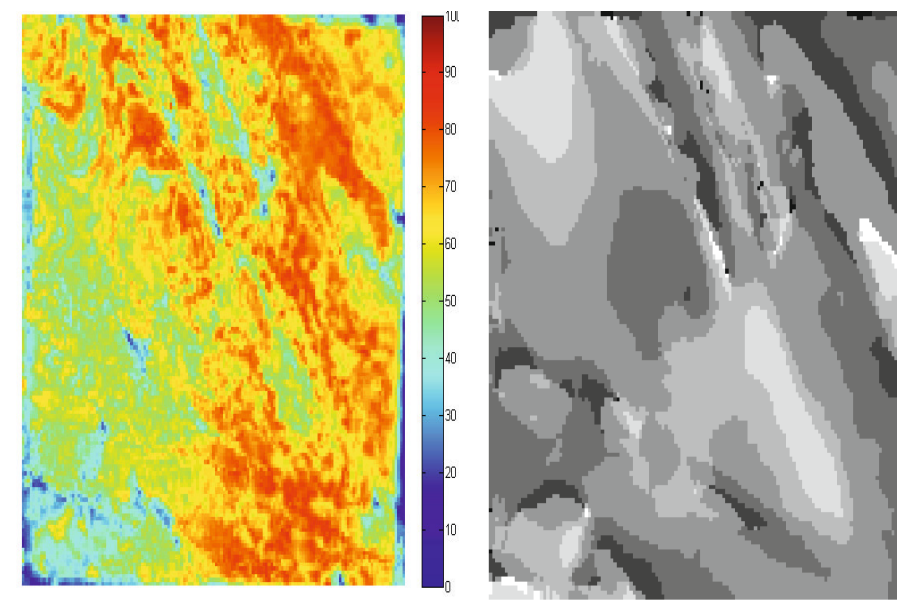

Relative orientations

of hexagonal lattice

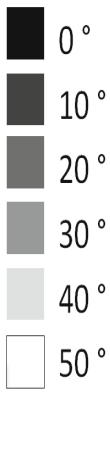

Fig. 11. Resulting image after subdivision of images from Figure 9 following the Macrofixe ${ }^{\circledR}$ method. Grain morphology (left) and hexagonal lattice relative orientations (right).

\section{$5 \quad$ Conclusion and Perspectives}

The recent advances made by CEA LITEN in the field of image processing and vision for the control and qualification of hexagonal-close-packed assembly of spherical microparticles have been presented. These control methods, called Microfixe ${ }^{\circledR}$ and Macrofixe $\AA$, are able to give significant information respectively at microscopic and 
macroscopic scales. From SEM or optical microscope images, using Delaunay triangulation, parameters such as surface ratio, number of grains, number of orientations and values of orientation can be extracted. From images taken by camera and thresholded to obtain binary images, the morphology of the grains at the macroscopic scale can be mapped. Useful information (mapping and orientation of the grains) is obtained first by processing six images of a sample illuminated under six different incidence angles separated by $10^{\circ}$ in the plane $\mathrm{xOy}$. For each image, the percentage of white pixels is calculated and then compared. Depending on the differences obtained, it is possible to determine whether the observed zone is properly structured, diffusing or not determined. In the case where it is not determined, the six original images are divided into four (for example). The divided parts of the image are then analyzed using the same protocol: calculation of the white pixels percentages, or subdivision if the gap does not allow a definition of the quality of organization. Step by step, an artificial image of the particle deposit is constituted, giving precise information on the morphology of the grains and their orientations.

Microfixe ${ }^{\circledR}$ and Macrofixe ${ }^{\circledR}$ control methods are key points in order to provide an accurate control and enhancement of the deposit structure, providing assistance in the monitoring of the process for rigorous developments in the area of self-assembled materials. Future work will be aimed at developing a real time macroscopic analysis, in order to implement this control method system in an industrial equipment of particles deposition.

Acknowledgements. The present research was funded by the FP7 programme "Flexible Compression Injection Molding Platform for Multi-Scale Surface Structures (IMPRESS). To Mr Martin RANSOM and Mr Jean-Marie Becker (CPE Lyon), for guidance and adjustments.

\section{References}

1. Zhang, G., Wang, D.: Colloidal Lithography, The Art of Nanochemical Patterning. Chem. Asian J 4, 236-245 (2009)

2. Ye, X., Qi, L.: Two-dimensionally patterned nanostructures based on monolayer colloidal crystals: Controllable fabrication, assembly, and applications. Nano Today 6, 608-631 (2011)

3. Chouquet, C., Gavillet, J., Ducros, C., Sanchette, F.: Effect of DLC surface texturing on friction and wear during lubricated sliding. Materials Chemistry and Physics 123, 367-371 (2010)

4. Chen, J.-K., Qui, J.-Q., Fan, S.-K., Kuo, S.-W., Ko, F.-H., Chu, C.-W., Chang, F.-C.: Using colloid lithography to fabricate silicon nanopillar arrays on silicon substrates. Journal of Colloid and Interface Science 367, 40-48 (2012)

5. Moon, G.D., Lee, T.I., Kim, B., Chae, G., Kim, J., Kim, S., Myoung, J.-M., Jeong, U.: Assembled Monolayers of Hydrophilic Particles on Water Surfaces. ACS Nano 5(11), 8600-8612 (2011) 
6. Retsch, M., Zhou, Z., Rivera, S., Kappl, M., Zhao, X.S., Jonas, U., Li, Q.: Fabrication of Large-Area, Transferable Colloidal Monolayers Utilizing Self-Assembly at the Air/Water Interface, Macromol. Chem. Phys. 210, 230-241 (2009)

7. Hillebrand, R., Muller, F., Schwirn, K., Lee, W., Steinhart, M.: Quantitative analysis of the grain morphology in self-assembled hexagonal lattices. ACS Nano 2(5), 913-920 (2008)

8. Acharya, S., Hill, J.P., Ariga, K.: Soft Langmuir-Blodgett Technique for HardNanomaterials. Adv. Mater 21, 2959-2981 (2009)

9. Bardosova, M., Pemble, M.E., Povey, I.M., Tredgold, R.H.: The Langmuir-Blodgett Approach to Making Colloidal Photonic Crystals from Silica Spheres. Adv. Mater. 22, 3104-3124 (2010)

10. Pan, F., Zhang, J., Cai, C., Wang, T.: Rapid Fabrication of Large-Area Colloidal Crystal Monolayers by a Vortical Surface Method. Langmuir 22(17), 7101-7104 (2006)

11. Zhang, Y.J., Li, W., Chen, K.J.: Application of two-dimensional polystyrene arrays in the fabrication of ordered silicon pillars. Journal of Alloys and Compounds 450, 512-516 (2008)

12. Ninove, F.-P.: Thesis from Ecole centrale de Lyon (France), Texturation de surface par laser femtoseconde en régime élastohydrodynamique et limite, tel-00688051, version 1 , ECL 2011-43 (2011)

13. Chouquet, C.: Thesis from l'institut National Polytechnique de Lorraine, Elaboration et caractérisation de revêtements type «Diamond-Like Carbon »déposés par un procédé chimique en phase vapeur assisté par un plasma basse fréquence (2008)

14. CEA LITEN, internal results, contact: olivier.dellea@cea.fr

15. Cocquerez, J.-P., Philipp, S.: Analyse d'images: Filtrage et segmentation, ISBN 2-22584923-4. (1995) Masson (ed.)

16. Otsu, N.: A threshold Selection Method from Gray-Level Histograms. IEEE Transactions on Sytems, Man, and Cybernetics smc-9 (1979)

17. Finkel, R.A., Bentley, J.L.: Quad Trees A Data Structure for Retrieval on Composite Keys. Acta Informatica 4, 1-9 (1974) 